\title{
Bölgesel iyonosferik Düşey Toplam Elektron İçeriğinin (VTEC) mekansal ve zamansal boyutlarda Çok Değişkenli Uyabilen B-Spline Regresyonu (BMARS) kullanılarak belirlenmesi ${ }^{* *}$
}

\author{
Mahmut Onur Karslıoğlu ${ }^{1,2, *}$, Murat Durmaz ${ }^{2}$ \\ 1 Orta Doğu Teknik Üniversitesi, Inşaat Mühendisliği Bölümü, Geomatik Mühendisliği Anabilim Dalı, Ankara, Türkiye \\ 2 Orta Doğu Teknik Üniversitesi, Jeodezi ve Coğrafi Bilgi Teknolojileri Bölümü, Ankara, Türkiye
}

\section{Özet}

Web Yayın: 10 Temmuz 2012 Mekansal ve zamansal bölgesel İyonosferin Düşey Toplam Elektron İçeriği (VTEC) cinsinden modellenmesi parametrik olmayan Çok Değişkenli Uyabilen B-Spline fonksiyonlarına Dayalı Regresyon (BMARS) kullanı-

Cilt: 1

Sayı: 1

Sayfa: 55 - 64

Mayıs 2012 larak gerçekleştirilmektedir. Gözlemlerden otomatik olarak üretilen Baz Fonksiyonları, karesel (ikinci derece) B-Spline fonksiyonlarının sıkılaştırılmış destekli tensör çarpımlarından oluşturulmaktadır. Yumuşatılmış bir yaklaştırıma sıralı ölçeklendirmeye dayalı bir model kurma stratejisiyle ulaşılmaktadır. Bu strateji veriye her ölçekte en iyi uyan B-Spline fonksiyonunu aramaktadır. İslenen veri grubu Avrupa' daki yersel GPS istasyonlarından toplanmış olup, 15 Şubat 2011 tarihinde meydana gelen jeomanyetik bir firtınayı da içermektedir. BMARS modellemesinin sonucu bu yöntemin etkinliğini ve potansiyelini açıkca göstermektedir. Hesaplanan sonuç, aynı zamanda gerek nümerik gerekse görsel olarak, tanınmış küresel ve bölgesel modellerle karşılaştırllmıştır. Küresel model küresel harmonik fonksiyonlara, bölgesel model de B-Spline fonksiyonlarina dayanmaktadir.

Anahtar Sözcükler

İyonosfer, GPS, MARS, B-spline, BMARS, Bölgesel modelleme

\section{Abstract \\ Regional spatio - temporal modeling of the ionospheric Vertical Total Electron Content (VTEC) using Multivariate Adaptive Regression B-Splines (BMARS)}

Pub. Online: 10 July 2012

Volume: 1

Number: 1

Page: $55-64$

May 2012

\begin{abstract}
Spatio-temporal Regional modeling of the ionosphere in terms of the vertical total electron content (VTEC) is accomplished using a non-parametric Multivariate Adaptive Regression B-Spline (BMARS) algorithm on the basis of Global Positioning System (GPS) observations. The basis functions are constructed as compactly supported tensor products of quadratic B-Splines which are derived from the observations automatically. A smooth approximation is achieved by scale-by-scale model building strategy which searches for best fitting $B$ Spline to the data at each scale. The real data set processed is gathered from ground based GPS stations in Europe and falls within the time interval of the geomagnetic storm on 15 February, 2011. The result of BMARS modeling apparently demonstrates the efficiency and the potential of the method. It is also compared both numerically and visually with a well-known global and regional VTEC modeling based on spherical harmonics and B-Splines respectively.
\end{abstract}

Keywords

Ionosphere, GPS, MARS, B-spline, BMARS, Regional modeling

\footnotetext{
$* *$

Hakemli değerlendirme sürecinden geçerek, Jeodezi ve Jeoinformasyon Dergisi'nde yayına kabul edilen ve asıl yazı dili İngilizce olan "Regional spatio - temporal modeling of the ionospheric Vertical Total Electron Content (VTEC) using Multivariate Adaptive Regression B-Splines (BMARS)" başılıkı eserin Türkçe diline çevirisidir. Çeviren; Mahmut Onur Karslıoğlu.
} 


\section{Giriş}

Yersel ve uzaysal platformlara yerleştirilmiş çift frekanslı GPS alıcılarından elde edilen gözlemler İyonosferin Toplam Elektron İçeriğinin (TEC) belirlenmesinde yaygın olarak kullanılmaktadır. TEC dağılımı radyo dalgalarının iyonlaştırılmış atmosfer içerisindeki yayılımını ciddi bir şekilde etkilemektedir. Bu durum GNSS ve uydu navigasyon (seyrüsefer) sistemlerini de içeren yer ve uzay bazlı iletişimin sağlanması açısından çok önemlidir (Liu ve Gao 2004; Policy Workshop Report 2011).

Eğik Toplam Elektron İçeriği (STEC - Slant Total Elektron Content) uydu ile alıcısı arasındaki sinyal yolu boyunca $1 \mathrm{~m}^{2}$ kesit alanına sahip kolon içerisindeki toplam elektron elektron yoğunluğunun integre edilmesiyle hesaplanmaktadır. Bu çalışmada ise modelleme için Dikey Toplam Elektron İçeriği (VTEC - Vertical Total Electron Content) tercih edilmektedir. Çünkü yersel GPS istasyonlarından toplanan gözlemler iyonosferin radyal geometrisine karşı daha düşük duyarlılık göstermektedir. Bu yaklaşımda, STEC'i VTEC'e çeviren eşleme fonksiyonundan kaynaklanan hataya ragmen, iyonosfer sonsuz küçük kalınlıkta ve belirli bir yükseklikteki küresel bir katman tarafından temsil edilmektedir ve bütün elektronların bu katmanda yoğunlaştığ1 varsay1lmaktadır. (Dettmering 2003; Jin vd. 2006, 2008; Brunini vd. 2010).

VTEC'in mekansal ve zamansal modellemesi coğrafik enlem, coğrafik boylam ve zamana bağlı olarak üç boyutta yapilabilmektedir (Mannucci vd. 1998; Hernandez-Pajares and Sanz 1999; Schaer 1999; Yuan and Ou 2002; Brunini vd. 2004; Jin vd. 2004). DTEI' nin coğrafi enlem ve boylam kullanılarak iki boyutta modellenmesi geleneksel olarak küresel fonksiyon sınıfinda olan küresel harmonik fonksiyonlarca yapılmaktadır. $\mathrm{Bu}$ anlamda kullanılacak verinin tüm küre üzerinde düzgün olarak dağılması gerekmektedir.

Bölgesel ve yerel elektron yoğunluğunun ve VTEC'in iyonosferin mekansal ve zamansal değişimleri için modellenmesi durumunda, Öklidan karesel (ikinci derece) B-Spline ve tensor çarpımlarına dayandırılan çok boyutlu yaklaşım, küresel fonksiyonların yerine Schmidt (2007), Schmidt vd. (2007a,b), Zeilhofer (2008) ve Nohutcu vd. (2007, 2010) tarafindan tercih edilmektedir. Çok boyutlu yaklaşımın detayları Garcia-Fernandez (2004) de bulunabilir. Farklı bir tür bölgesel ve yerel modelleme, parametrik olmayan ve parça parça doğrusal fonksiyonlardan oluşan Çok Değiş̧kenli Uyabilen Spline Regresyonu (MARS) ile de yapılabilmektedir.

MARS çok büyük ölçüm setlerini işleyebilmekte ve fark1 zaman serilerinde doğrusal ve doğrusal olmayan modeler için kullanılabilmektedir (Lewis and Stevens 1991; Ekman ve Kubin 1999; Yang vd. 2004; Crino ve Brown, 2007). MARS veriye uyabilen bir yöntem olup 1zgaralama gerektirmemektedir. Durmaz vd. (2010) MARS modelini bölgesel DTEİ modellenmesi için Avrupa üzerindeki büyük bir alana başarıyla uygulamıştır. Bunun akabinde, gözlem noktalarında oluşturulan tensor çarpımı B-Spline baz fonksiyonlarına dayalı Çok Değişkenli Uyabilen B-Spline Regresyon (BMARS) Durmaz ve Karslioglu (2011) tarafindan yayınlanmıştır. MARS ve BMARS arasındaki farklılık, sadece farklı baz fonksiyonlarını kullanmalarıyla sınırlı değildir. BMARS ayrıca sıralı ölçeklendirmeye dayalı bir model stratejisini kullanmaktadır. Burada baz fonksiyonları büyük ölçekten küçük ölçeğe doğru seçilmektedir. Bu yaklaşım sayesinde baz fonksiyonlarının miktarında anlamlı bir indirgemeye gidilmekte ve böylece original MARS'dan daha yumuşak bir modellemeye ulaşılmaktadır. B-Spline baz fonksiyonlarının sıkıştırılmış destekli olma özellikleri nedeniyle küçük ölçekler için daha iyi yerelleştirme yapılabilmektedir (Bakin vd. 1997; Bakin vd. 2000).

Bu çalışmada BMARS, 15 Şubat 2011 tarihindeki jeomanyetik firtınayı içeren Avrupa üzerindeki verilere bölgesel VTEC'i modellemek amacıyla uygulanmıştır. Algoritmanın performansını değerlendirebilmek için BMARS sonuçları hem nümerik hem de görsel olarak Nohutcu vd. (2010) tarafindan sunulan B-Spline'a dayalı diğer bir bölgesel iyonosfer modeli ve küresel harmoniklere dayalı bir modelleme ile karşılaştırılmaktadır.

Makalenin bölümleri aşağıdaki gibi düzenlenmiștir: İkinci bölümde yersel GPS istasyonlarındaki gözlemlerden VTEC'in nasıl hesaplandığı verilmektedir. Üçüncü bölüm BMARS'ın matematiksel temellerini anlatmaktadır. Dördüncü bölüm Avrupa üzerindeki uygulamanın sonuçlarını ve onların analizini göstermektedir ve farklı VTEC modelleme stratejilerinin karşılaştırılmasına yoğunlaşmaktadır. Son bölümde ise sonuç sunulmaktadır.

\section{Yersel GPS gözlemlerinden VTEC modellemesi}

Elektromanyetik sinyalin iyonosfer kaynaklı mesafe gecikmesi, STEC'in ve sinyalin frekansının bir fonksiyonudur. Mesafe gecikmesi $I_{i}$ metre cinsinden aşağıda gibi ifade edilebilmektedir.

$I_{i}= \pm \frac{40.3}{f_{i}^{2}} S T E C$

burada $f_{i}, L_{i}, i=1,2$ taşıyıcı sinyalinin frekansıdır (Liu ve Gao 2003; Hofmann-Wellenhof vd. 2008). Sinyaldeki gecikme kod gözlemleri için pozitif, taşıyıcı faz gözlemleri için ise negatifdir.

Geometriden bağımsız lineer kombinasyon diye adlandır1lan iyonosferik gözlem kod ya da faz ölçümlerinin aynı anda alınan farklarından oluşturulmaktadır. $\mathrm{Bu}$ kombinasyonun kullanılmasıyla uydu ile alıcı arasındaki geometrik mesafe ile frekansdan bağımsız bias'ın ortadan kaldırılması mümkün olmaktadır (Ciraolo vd. 2007). Pseude kod ölçümleri için iyonosferik gözlem, $P_{4}$ aşağıdaki gibi gösterilmektedir:

$P_{4}=P_{1}-P_{2}=I_{1}-I_{2}+b r+b s+\varepsilon_{p}$

burada $P_{1}, L_{1}$ frekansı için, pseude mesafe, $b r$ ve $b s$ pseude mesafe için alıcı ve uydunun donanımlarından kaynaklanan frekanslar arası bias ya da gecikme (IFB) ve $\varepsilon_{p}$ ise $P_{1}$ ve $P_{2}$ gözlemlerinin ratgele gürültüleri ve multi-path etkilerinden oluşan toplam bir hatadır. Faz ölçmeleri için iyonosferik gözlem, $\Phi_{4}$ aşağıdaki gibi yazılabilmektedir: 
$\Phi_{4}=\Phi_{1}-\Phi_{2}=L_{2}-L_{1}+\lambda_{1} N_{1}-\lambda_{2} N_{2}+B r+B s+\varepsilon_{L}$

burada $\Phi_{i}, L_{i}$ için metre cinsinden taşıyıcı faz gözlemi, $\lambda_{i} L_{i}$ 'nin dalga boyu, $N_{i}$ tam sayı taşıyıcı faz bilinmeyeni, $B r$ ve $B s$ taşıyıcı faz ölçümlerine ait alıcı ve uydunun frekanslar arası bias' larıdır (IFB). $\varepsilon_{L}, \Phi_{1}$ ve $\Phi_{2}$ deki gözlemsel gürültü ve birden fazla yol hatalarının kombinasyonudur. STEC, (1) numaralı ve (2) numaralı ya da 3 numaralı denklemlerin birleştirilmesiyle hesaplanabilmektedir. Taşıyıcı faz ölçmeleri, kod ölçmeleriyle karşılaştırıldığında daha düşük gürültü içermelerine rağmen, ön işlemedeki tamsayı faz bilinmeyeninin kestirilmesi zorunluluğu nedeniyle, burada STEC hesaplanması için tercih edilmemektedir. Bunun yerine taşıyıcı faz tarafından yumuşatılan kod ölçümleri (taşıyıcı faz nivelmanı) kullanılmaktadır. Bu sayede hem gürültünün hem de tamsayı faz bilinmeyenlerinin etkisi azaltılmaktadır (Ciraolo vd. 2007). Bu işlem (2) ve (3) numaralı denklemlerin aşağıda gösterildiği gibi eklenmesiyle başlamaktadır:

$P_{4}+\Phi_{4}=\lambda_{1} N_{1}-\lambda_{2} N_{2}+B r+B s+b r+b s+\varepsilon_{p}$

Burada $P_{4}$ ve $\Phi_{4}$, GPS gözlemlerinden elde edilmektedir. Tamsayı bilinmeyeni terimleri $N_{1}$ ve $N_{2}$ sinyal kayması içermeyen her sürekli taşıyıcı faz gözlem aralığında sabittir.

IFB'lar günlük hatta aylık stabil olarak değerlendirilebilir ve böylece sürekli bir gözlem aralığı için sabit olarak alınabilmektedir (Gao vd. 1994). (4) numaralı denklemde taşıyıcı faz gözlemlerine ait olan gürültü ve birden fazla yol terimi ihmal edilmektedir. Çünkü her iki terim de kod gözlemlerindeki değerlerinden daha düşük etkiye sahiptirler. Buradan her sürekli gözlem aralığı için ortalama bir değer, $\left\langle P_{4}+\Phi_{4}\right\rangle_{\text {arc }}$ hesaplanabilmektedir:

$$
\begin{aligned}
\left\langle P_{4}+\Phi_{4}\right\rangle_{\mathrm{arc}}= & \frac{1}{n} \sum_{i=1}^{n}\left(P_{4}+\Phi_{4}\right)_{i}=\left\langle\lambda_{1} N_{1}-\lambda_{2} N_{2}\right\rangle_{\mathrm{arc}} \\
& +B r+B s+b r+b s+\left\langle\varepsilon_{P}\right\rangle_{\mathrm{arc}}
\end{aligned}
$$

burada $n$ her sürekli gözlem aralığındaki gözlem sayısıdır. (3) numaralı denklem (5) numaralı denklemden çıkarıldığında tamsayı bilinmeyeni ortadan kaldırılmaktadır:

$$
\tilde{P}_{4}=\left\langle P_{4}+\Phi_{4}\right\rangle_{\mathrm{arc}}-\Phi_{4} \approx I_{1}-I_{2}+b r+b s+\left\langle\varepsilon_{P}\right\rangle_{\mathrm{arc}}-\varepsilon_{L}
$$

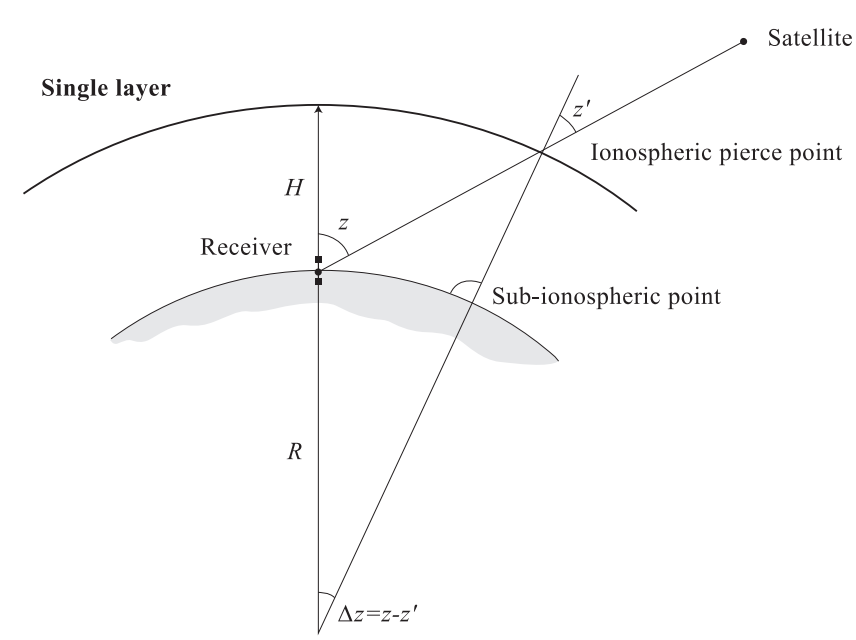

Şekil 1: lyonosfer için tek tabaka modeli (Schaer, 1999) burada $\tilde{P}_{4}$ taşıyıcı faz tarafindan yumuşatılmış kod iyonosferik gözlemidir. Denklem (1) deki iyonosferik mesafe gecikmesi (6) numaralı denkleme yerleştirilirse, STEC, TECU cinsinden aşağıdaki gibi hesaplanabilmektedir:

$$
S T E C=\left(\tilde{P}_{4}-b_{r}-b_{s}-\left\langle\varepsilon_{P}\right\rangle_{\mathrm{arc}}+\varepsilon_{L}\right) \frac{\left(f_{1}^{2} f_{2}^{2}\right)}{40.3\left(f_{2}^{2}-f_{1}^{2}\right)}
$$

Yükseklikten bağımsız iki boyutlu ya da zamanın üçüncü boyut olarak alındığı üç boyutlu iyonosfer modelleri için STEC, tek tabaka modeli kapsamında çoğunlukla bir eşleme fonksiyonu kullanılarak VTEC' e dönüştürülmektedir. Tek tabaka modeli tüm elektronların sonderece ince kalınlıktaki küresel bir tabakada toplandığı varsayımından yola çıkmaktadır (Şekil 1). STEC ve VTEC ilişkisini kuran tek tabaka eşleme fonksiyonu aşağıdaki gibi verilmektedir:

$$
F_{I}=\frac{S T E C}{V T E C}=\frac{1}{\cos z^{\prime}} \text { ile } \sin z^{\prime}=\frac{R}{R+H} \sin z
$$

burada $R$ yeryuvarının ortalama yarıçapı, $z$ ve $z^{\prime}$ uydunun gözlem noktasında ve iyonosfer kesme noktasındaki zenit açılarıdır. $H$, idealize edilmiş tabakanın yüksekliği ya da ortalama yükseklikdir (Şekil 1). Bu yükseklik yaklaşık olarak maksimum elektron yoğunluğuna tekabül etmektedir ve yüksekliği 350 ile $450 \mathrm{~km}$ arasında değişmektedir (Schaer 1999; Seeber 2003; Hofmann-Wellenhof vd. 2008).

\section{3. Çok Değişkenli Uyabilen B-Spline Regresyonu (BMARS)}

Çok Değişkenli Uyabilen B-Spline Regresyonu yani BMARS algoritması orijinalı olan Çok Değişkenli Uyabilen Spline Regresyonuyla (MARS) benzer prensiplere dayanmaktadır (Friedman 1991). Sadece kesilmiş güç baz fonksiyonlarının kullanıldığı MARS'a karşın BMARS, baz fonksiyonları olarak herhangi bir dereceden B-Spline fonksiyonlarının getirdiği avantajlardan faydalanmaktadır (Bakin vd. 1997; Bakin vd. 2000). B-Spline fonksiyonları sıkılaştırılmış destekli fonksiyonlardır. $\mathrm{Bu}$ ise fonksiyon değerlerinin sıkılaştırılan intervalin dışında sıfır değerini aldığ

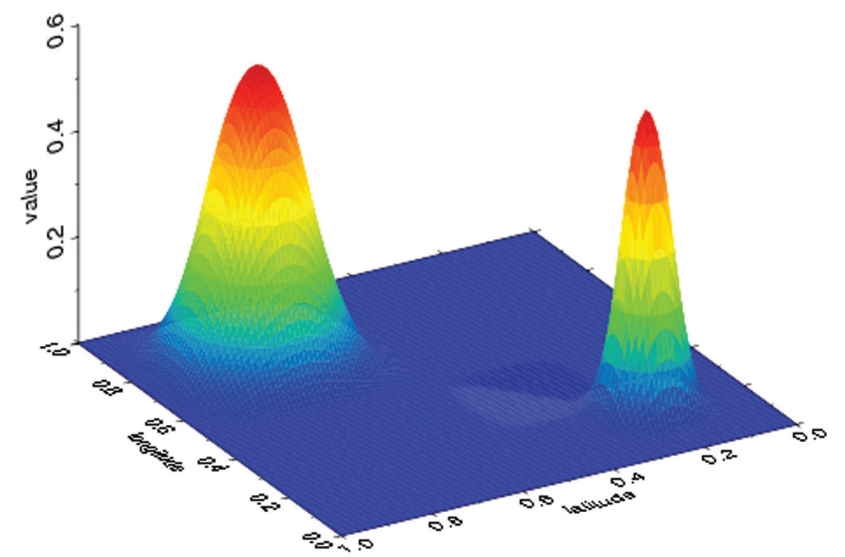

Şekil 2: Düzey ya da kademe 2 ve 3 için karesel B-Spline Ölçek Fonksiyonları. Düzey yükseldikçe fonksiyonların tanımlandığı aralık daralmaktadır. 
Örnek olarak Şekil 2 de ölçek 2 ve 3 için iki boyutlu karesel B-Spline fonksiyonunun ölçek fonksiyonları görülmektedir. Desteklenen intervalin ya da aralığın uzunluğu seçilen düğüm noktaları tarafindan belirlenmektedir. Bu durum algoritmayı sıralı ölçeklendirme yaklaşımına dayalı yeni çeşit bir model yapılandırmasına götürmektedir. Kullanılan farklı baz fonksiyonlarının çeşiti dışında, MARS ve BMARS arasındaki diğer bir fark da işte bu farklı model kurma yapılandırması ya da stratejisine dayanmaktadır. Burada ölçek, destek aralığının uzunluğuna karşılık gelmektedir.

$\boldsymbol{y}$ vektörü $\boldsymbol{x}_{k}=\left[x_{k, 1}, x_{k, 2}, \ldots, x_{k, p}\right]^{T}, k=1,2, \ldots, N$ konumunda, $N$ sayıdaki gözlemleri içeren gözlem vektörü olsun. Burada konumun boyutudur. Verilen tanımlardan yola çıkarak aşağıdaki gözlem denklemi oluşturulabilmektedir

$$
\boldsymbol{y}=\left[\begin{array}{c}
y_{1} \\
y_{2} \\
\vdots \\
y_{k} \\
\vdots \\
y N
\end{array}\right]=\left[\begin{array}{c}
f\left(\boldsymbol{x}_{1}\right) \\
f\left(\boldsymbol{x}_{2}\right) \\
\vdots \\
f\left(\boldsymbol{x}_{k}\right) \\
\vdots \\
f\left(\boldsymbol{x}_{N}\right)
\end{array}\right]+\left[\begin{array}{c}
e_{1} \\
e_{2} \\
\vdots \\
e_{k} \\
\vdots \\
e_{N}
\end{array}\right]
$$

burada $f(\boldsymbol{x})$ bilinmeyen çok değişkenli fonksiyon, $\boldsymbol{e}=\left[e_{1}, e_{2}, \ldots, e_{N}\right]^{T}$ ortalaması sıfir ve sonlu varyanslı gözlem hatalarıdır. Gözlemlere en iyi uyarak $f(\boldsymbol{x})^{\prime}$ 'e yaklaşan değer $\hat{f}(\boldsymbol{x})$ en küçük kareler regresyonuyla bulunmaktadır (Durmaz ve Karslioglu 2011). BMARS algoritması aşağıdaki regresyon fonksiyonu, $f(\boldsymbol{x})^{\prime} \mathrm{i}$ en iyi uyan baz fonksiyonları $h_{i}(\boldsymbol{x})$ 'i otomatik olarak üreterek kurmaktadır

$$
f(\boldsymbol{x})=\sum_{i=0}^{M-1} \beta_{i} h_{i}(\boldsymbol{x})
$$

(10) numaralı denklemde, $M$ baz fonksiyonlarının sayısı, $\beta_{i}$ de baz fonksiyonlarının katsayılarıdır. Baz fonksiyonları aşağıdaki gibi yeniden tanımlanabilirler

$$
h_{i}(\boldsymbol{x})=\prod_{l=1}^{L_{i}} B_{d, t_{l, i}}\left(\boldsymbol{x}_{v(l, i)}\right) \quad \text { ile } \quad i>0
$$

burada $L_{i}, i^{\text {th }}$ baz fonksiyonunun etkileşim derecesi, $v(l, i)$ B-Spline'a ait ilgili düğüm noktası $t_{l, i}$ ile ilintili değişkendir. Çok değişkenli B-Spline baz fonksiyonları, $h_{i}(\boldsymbol{x})$ tek değişkenli B-Spline fonksiyonlarının tensor çarpımlarından düzenlenmektedir. Bu şekilde elde edilmiş baz fonksiyonlarının kullanabilmesi için model oluşturulması sürecinde ve baz fonksiyonlarının yapılandırılmasında küçük değişikliklere gidilmelidir. İlk olarak BMARS algoritması regresyon fonksiyonunu aynı MARS da olduğu gibi iki aşamada yani ileriye ve geriye doğru şeklinde oluşturmaktadır. İleriye Doğru Aşama'da veriye ihtimal dahilinde fazla uyan oldukça geniş bir model üretilmektedir. Geriye Doğru Aşama'da ya da eleme aşamasında ise düzeltmelere en az etki eden yetersiz baz fonksiyonları modelden atılmaktadır. Böylece de uyumun kalitesi arttırılmaktadır. Bu da güncellenen modelin tahmin hatası olarak Genelleştirilmiş Çapraz Doğrulamanın (GCV) minimum yapılması anlamına gelmektedir. B-Spline baz fonksiyonları düğüm noktaları konumları, $C_{s}^{j}$ nin iç içe yuvalanmış gruplarından üretilmektedir. Burada $s$ çok değişkenli değişken $\boldsymbol{x}$ 'in ölçek indeksi ve $j$ bileşen indeksi olarak tanımlanmaktadır. Düğüm noktası konumları her değişken, $x_{j}$ için $x_{k, j}<x_{k+1, j}, k=1,2, \ldots, N-1$ şeklinde artan bir sırada düzenlenmiştir. Burada $N$ gözlemlerin sayısıdır. O zaman, $C_{s}^{j}$ grubu aşağıdaki gibi tanımlanmaktadır ve

$$
\begin{aligned}
C_{s}^{j} & =\left\{x_{1, j}, t_{r, j}^{s}, x_{N, j}\right\}, \quad r=1,2, \ldots, 2^{s}-1 \mathrm{ve} \\
t_{r, j}^{s} & =x_{\left[r 2^{-s} N+0.5\right], j}
\end{aligned}
$$

Burada yuvarlanmış tam sayılar, $\left[r 2^{-s} N+0.5\right]$ düzenlenmiş düğüm noktası konumları $x_{k, j}$ 'nın yüzdesel değeri $\mathrm{r} \times 2^{-\mathrm{s}} \times 100$ 'nin kademesine karş1lık gelmektedir (Durmaz ve Karslioglu 2011; Bakin vd. 1997). Örneğin, Ölçek indeksi $s=1$ için grup, $C_{1}^{j},\left\{x_{1, j}, x_{[N / 2+0.5], j}, x_{N, j}\right\}$ olarak tanımlanmaktadır (Durmaz ve Karslioglu 2011). $s=2$ için, $C_{2}^{j}=\left\{x_{1, j}, x_{[N / 4+0.5], j}\right.$, $\left.x_{[2 N / 4+0.5], j}, x_{[3 N / 4+0.5], j}, x_{N, j}\right\}$ içindir. Ölçek indeksi $s$ büyüdükçe, B-Spline'nın ölçeği (destek aralığı) azalmaktadır. Bu da daha fazla lokalize eden, yerelleştiren fonksiyonların oluşmasıyla sonuçlanmaktadır. Her grup $C_{s}^{j}$ için sonlu nokta enterpolasyonu kullanılarak farklı bir B-Spline grubu üretilmektedir. Sonlu nokta Enterpolasyonu interval ya da aralık sınırlarındaki ilk iki ve son iki ölçek fonksiyonunu değiştirmektedir (Durmaz ve Karslioglu 2011).

\section{1 İleriye Doğru Aşama (Adım)}

İleriye Doğru Aşama sabit bir baz fonksiyonu $h_{0}(\boldsymbol{x})=1$ 'le başlamakta ve onun katsayılarını en küçük kareler kestirim yöntemine göre kestirmektedir. Daha sonra ölçek indeksi $s=1$ 'le başlayan modele tensor çarpımlarından üretilen yeni baz fonksiyonları eklenmektedir. Yeni tensor üretimi B-Spline, $h_{m}(\boldsymbol{x})$ her iterasyonda, $C_{s}^{j}$ düğüm noktası konum setlerinden tanımlanan tüm mevcut tek değişkenli B-Spline baz fonksiyonları arasından aranmaktadır:

$h_{m}(\boldsymbol{x})=h_{i}(\boldsymbol{x}) B_{d, t_{n, j}^{s}}\left(x_{j}\right)$

Burada $h_{i}(\boldsymbol{x}), \quad(0 \leq i<m)$ modelde önceden seçilmiş baz fonksiyonu ve $B_{d, t_{n, j}^{s}}\left(x_{j}\right), d$ dereceden tek değişkenli B-Spline fonksiyonudur. $B_{d, t_{n, j}^{s}}\left(x_{j}\right), x_{j}$ değişkeni ile ilişkilendirilen $C_{s}^{j}$ grubuna ait düğ̈üm noktası konumu $t_{n, j}^{s}$ den oluşturulmaktadır. Bu durum $h_{i}(\boldsymbol{x})$ fonksiyonunun $x_{j}$ değişkenini içermemesini gerektirmektedir. Düzeltmeleri (residualları) en fazla küçülten baz fonksiyonları $h_{m}(\boldsymbol{x})$ modele eklenmektedir (Bakin vd. 1997; Bakin vd. 2000). Eğer sonuçlanan modelin GCV değeri artmaya eğilim gösteriyorsa ölçek indeksi arttırılmakta ya da bir başka deyişle destek aralığ küçültülmektedir. Bu da algoritmanın modele daha önceden yeterli sayıda büyük ölçekli bileşenleri yerleştirdiği ve bu nedenle de daha küçük ölçekli bileşenlerin aranmak zorunda olduğu anlamına gelmektedir (Durmaz ve Karslioglu 2011). 


\subsection{Geriye Doğru Aşama}

Geriye Doğru Aşama MARS algoritmasıyla benzerdir. İleriye Doğru Aşama'da genişletilen model düzeltmelere az katkı yapan terimlerin çıkarılmasıyla küçültülerek, sonuç modelin GCV değeri cinsinden iyi bir tahmin performansını yakalaması sağlanmaktadır. Bu iteratif süreç sonuç modelde aşağıda verilen GCV değerini minimize yapabilen optimal ve efektif bir terim sayısı bulununcaya kadar sürdürülmektedir:

$$
G C V(\lambda)=\frac{\sum_{k=1}^{N}\left[y_{k}-\sum_{i=0}^{M-1} \hat{\beta}_{i} h_{i}\left(\boldsymbol{x}_{k}\right)\right]^{2}}{(1-k(\lambda) / N)^{2}} .
$$

(14) numaralı denklemde, $\lambda$ aynı zamanda regularizasyon parametresi gibi de algılanabilen bir ayarlama parametresi, $K(\lambda)$ modeldeki efektif terim sayısının ölçütüdür ve basitçe modeldeki terim sayılarının lineer fonksiyonu olarak tanımlanabilmektedir.

BMARS algoritması sıralı ölçeklendirme yaklaşımına dayalı model stratejisini ((13) numaralı denklemde bir ölçekten diğer ölçeğe geçiş) kullanmaktadır. Bu strateji sayesinde küçük $s$ ler için ya da büyük ölçekler için aranacak olan aday baz fonksiyonlarının sayısı anlamlı bir şekilde küçültülmektedir. Algoritmanın diğer bir avantajı ise küçük ölçek özelliklerini büyük ölçek özelliklerinden ayırması ve böylece daha yumuşak bir kestirim fonksiyonuna ulaşmasıdır (Bakin vd. 1997).

\section{BMARS Kullanılarak Bölgesel lyonosfer Modellemesi}

VTEC modellenmesi için (9) numaralı gözlem denklemi aşağıdaki şekilde yeniden düzenlenmektedir

$$
\left[\begin{array}{c}
y_{1} \\
y_{2} \\
\vdots \\
y_{k} \\
\vdots \\
y_{N}
\end{array}\right]=\left[\begin{array}{c}
\operatorname{VTEC}\left(\boldsymbol{x}_{1}\right) \\
\operatorname{VTEC}\left(\boldsymbol{x}_{2}\right) \\
\vdots \\
\operatorname{VTEC}\left(\boldsymbol{x}_{k}\right) \\
\vdots \\
\operatorname{VTEC}\left(\boldsymbol{x}_{N}\right)
\end{array}\right]+\left[\begin{array}{c}
e_{1} \\
e_{2} \\
\vdots \\
e_{k} \\
\vdots \\
e_{N}
\end{array}\right]
$$

burada $\operatorname{VTEC}(\boldsymbol{x})$, BMARS algoritmasının 3D olarak yere bağlı referans sisteminde kestirdiği VTEC model fonksiyonu, $x_{k}=[\lambda, \varphi, t]^{\mathrm{T}}$ konum, $\lambda$ coğrafi boylam, $\varphi$ coğrafi enlem, $t \boldsymbol{U} \boldsymbol{T}$ "Universal Time" cinsinden zamandır. $N$, VTEC gözlemlerinin sayısını, $y_{k}, x_{k}=\left[\lambda_{k} \varphi_{k} t_{k}\right]^{T}, k=1,2, \ldots, N$ konumundaki $k^{\text {th }}$ VTEC gözlemini, $e_{k}$ rastgele gözlem hatalarını temsil etmektedir. Bu çalışmadaki BMARS modellleri iyi nümerik özelliklerinden ötürü ikinci derece ya da karesel B-Spline fonksiyonlarından baz fonksiyonları olarak yararlanmaktadir. Nohutcu vd. (2010) ve Schmidt vd. (2007a, b) tarafindan da gösterildiği gibi karesel B-Spline fonksiyonları iyonosfer modellenmesinde yaygın olarak kullanılmaktadır. Daha iyi yorumlanabilmesi için, sonuçta oluşan BMARS modeli ((15) numaralı denkleme bakınız) aşağıda verilen açık biçimde yazılmıştır:

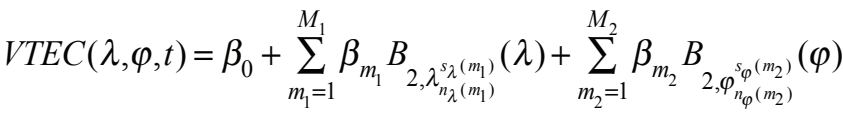

$$
\begin{aligned}
& +\sum_{m_{3}=1}^{M_{3}} \beta_{m_{3}} B_{2, t_{n_{t}}^{s_{t}\left(m_{3}\right)}}(t)+\sum_{m_{4}=1}^{M_{4}} \beta_{m_{4}} B_{2, \lambda_{2}^{s_{2}\left(m_{4}\right)}}(\lambda) B_{2, \varphi_{n_{\lambda}\left(m_{4}\right)}^{s_{\varphi}\left(m_{4}\right)}}(\varphi) \\
& +\sum_{m_{5}=1}^{M_{5}} \beta_{m_{5}} B_{\substack{2, \lambda_{n_{\lambda}\left(m_{5}\right)}^{s_{\lambda}\left(m_{5}\right)} \\
n_{2, t}}}(\lambda) B_{\left.2, t_{t}^{s_{t}\left(m_{5}\right)}\right)}(t) \\
& +\sum_{m_{6}=1}^{M_{6}} \beta_{m_{6}} B_{2, t_{n_{t}\left(m_{6}\right)}^{s_{t}\left(m_{6}\right)}}(t) B_{2, \varphi_{n_{\varphi}\left(m_{6}\right)}^{s_{\varphi}\left(m_{6}\right)}}(\varphi) \\
& +\sum_{m_{7}=1}^{M_{7}} \beta_{m_{7}} B_{2, \lambda_{n_{\lambda}\left(m_{7}\right)}^{s_{\lambda}\left(m_{7}\right)}}(\lambda) B_{2, \varphi_{n_{\varphi}\left(m_{7}\right)}^{s_{\varphi}\left(m_{7}\right)}}(\varphi) B_{2, t_{n_{t}\left(m_{7}\right)}^{s_{t}\left(m_{7}\right)}}(t)
\end{aligned}
$$

burada terimlerin toplam sayısı, $M=1+\sum_{i=1}^{7} M_{i}$ olarak tanımlanmıştır. Bütün tek değişkenli B-Splines $B_{2, \lambda^{s_{\lambda}\left(m_{i}\right)}}(\lambda)$, $B_{2, \varphi_{n_{\varphi}\left(m_{i}\right)}^{s_{\varphi}\left(m_{i}\right)}}(\varphi)$ ve $B_{2, t_{n_{t}}^{s_{t}\left(m_{i}\right)}}(t)$, 3. Bölümde açıklandiğ gibi $C_{s_{\lambda}\left(m_{i}\right)}^{\lambda}, C_{s_{\varphi}\left(m_{i}\right)}^{\varphi}$ ve $C_{s_{t}\left(m_{i}\right)}^{t}$ gruplarından üretilmiş̧tir. Grupların içerisindeki $s_{\lambda}\left(m_{i}\right), s_{\varphi}\left(m_{i}\right)$ ve $s_{t}\left(m_{i}\right), m_{i}^{\text {th }}$ terimdeki $\lambda, \varphi$ ve $t$ değişkenlerine ait B-Spline ölçeklerini tanımlamaktadır. $\beta_{0}$ sabit fonksiyonun katsayısıdır, $\beta_{m i}$ ise ilgili tensörlerden üretilen B-Spline'ların katsayılarıdır. (16) numaralı denklemden de görüldüğü gibi, BMARS regresyon fonksiyonunu tensörlerden üretilmiş farklı ölçek ve etkileşimleri içeren B-Spline' lardan kurmaktadir.

$\mathrm{Bu}$ çalışmada yazarlar tarafindan geliştirilmiş ve kurulmuş MATLAB rutinleriyle üretilen BMARS algoritmaS1 X-sınıfındaki bir güneş patlamasından kaynaklanan 15 Şubat 2011 tarihindeki jeomanyetik fırtınanın Avrupa üzerinde ortaya çıkardığı VTEC dağılımının modellenmesine uygulanmaktadır. Bunun için iki veri grubu Uluslarası GNSS servisine (IGS) ve/veya (EUREF) ağına ait olan 29 yersel GPS istasyonundan 17 ve 18 Şubat 2011 günleri için toplanmaktadır. Bu zaman aralığı jeomanyetik fırtınadan şiddetle etkilenen VTEC dağılımını modelleyebilmek için kasıtlı olarak seçilmiştir. VTEC haritası üretmek amacıyla 24 saatlik bir veri grubundan 1 saatlik bir alt grup her gün için kullanılmaktadır. Her alt grup seçilmiş bölge için iyonosferin yüksek değişimlerinin olduğu yerel öğle saati 10:00 AM UT de merkezlendirilmiştir. Uydular için IFB değerleri "Center of Orbit Determination in Europe" (CODE) den alınmakta, alıcı IFB değerleri ise kendi yazılımımız tarafından kestirilmektedir.

TECU (1 Toplam Elektron İçeriği Birimi $\left(\right.$ TECU) $=10^{16}$ elektron $/ \mathrm{m}^{2}$ ) cinsinden VTEC değerleri her gözlem için Bölüm $2^{\prime}$ de de anlatıldığı gibi yükseklik kesme açısı $15^{\circ}$ ve tek tabakanın yüksekliği $400 \mathrm{~km}$ alınarak hesaplanmaktadır. Gözlemlerin eşit duyarlık ve kalitede olduğu kabul edilmektedir. Uydu konumları çeşitli IGS analiz merkezlerindeki hassas uydu koordinatlarını içeren dosyalardan interpolasyon yapılarak üretilmektedir.

Yere Bağlı sistemde karesel B-Spline baz fonksiyonlarını kullanan BMARS algoritmasıyla 17 Şubat 2011 günündeki 
S1 diye tanımlanan ilk alt veri grubundan üretilen VTEC haritası Şekil 3(a) da, 18 Şubat 2011 günündeki S2 diye tanımlanan ikinci alt veri grubundan üretilen VTEC haritası Șekil 3(b) de sunulmuştur.

Göz önüne alınan baz fonksiyonu sayısı (MBFA) 300 dür. Kestirilmiş gözlemlerle original gözlemlerin farklarından hesaplanan Karesel Ortalama Hatanın Karekökü (RMSE), S1 için 0.3825 TECU dur. Burada seçilmiş terimlerin sayısı 279 olup sonuçta oluşan ölçek indeksi 3 tür. 289 seçilmiş terim sayısı ve sonuçta oluşan ölçek indeksi 4 le, S2 için RMSE değeri 0.4601 TECU olarak hesaplanmıştır.

8. derece ve 8 . mertebeden $(n=8, m=8)$ Küresel Harmonik açılımlara dayalı yere bağlı referans sisteminde yapılan modellemenin sonucu Şekil (4a) ve (4b) de S1 ve S2 alt very grupları için verilmiştir. RMSE değeri S1 için 0.562 , $\mathrm{S} 2$ için 0.893 olarak ortaya çıkmıştır.

Şekil 5, yere bağlı referans sisteminde ikinci dereceden ya da normalize edilmiş karesel B-Spline kullanarak yapılan diğer bir bölgesel modellemenin sonucunu göstermektedir. BMARS'la uyumlu olması açısından B-Spline'nın düzeyi ya da kademesi ki bu BMARS'ta ölçeğe karşılık gelmektedir (Şekil 2 ye bakınız) her boyut $\lambda$, $\varphi$, ve $t$ için 3 alınmıştır. Bu algoritmada $1000\left(=\left[2^{3}+2\right]^{3}\right)$ tane katsayının hesaplanması anlamına gelmektedir. B-Spline'a dayalı VTEC modelinin RMSE değerleri S1 için 0.387 TECU (Şekil 5(a), S2 için 0.502 TECU (Şekil 5(b) dur. S1 veri grubunda büyük VTEC değişimlerinin bulunmaması nedeniyle, modelleme sonuçları RMSE değeri açısından bütün modellerde benzerdir.

Yine de, görsel karş1laştırma ve nümerik sonuçlar BMARS'ın küresel harmonik (SH) be B-Spline modellemelere göre daha küçük RMSE değerleriyle daha yumuşak VTEC haritaları ürettiğini göstermektedir. SH ve B-Spline modellemenin teorik ayrıntıları için Nohutcu vd. (2010) kaynak olarak verilmektedir. Jeomanyetik aktivitenin etkisi tüm VTEC haritalarında 30-35 TECU'ya kadar görülebilmektedir. Ayrıca SH, B-Spline ve BMARS modellemelerinin fark1, farkların karesel ortalamasının karekökü cinsinden RMS (Root Mean Square), VTEC fark haritaları şeklinde, Şekil 6, 7 ve 8 de sunulmuştur.

Şekil 6, BMARS ve SH modellemeleri arasındaki fark1, VTEC fark haritaları olarak göstermektedir. RMS değeri, $\mathrm{S} 1$ için, $\mathrm{RMS}=0.8251$ TECU ve $\mathrm{S} 2$ için, $\mathrm{RMS}=1.7009$ dur. Şekil 7 de ise B-Spline ve SH modellemesinin fark1, fark haritaları olarak ilgili RMS değerleriyle birlikte verilmektedir. Burada S1 için RMS=0.6516 TECU, S2 için RMS $=1.078$ TECU dur.

Son olarak VTEC fark haritaları RMS değerleriyle birlikte, BMARS ve B-Spline modellemelerinin farkını vurgulamak için üretilmiştir (Şekil 8). Buna göre S1 için RMS= 0.7675 TECU, S2 için RMS= 1.0215 TECU dur.

S1 veri grubundaki düşük değişimler nedeniyle tüm modelleme sonuçlarında küçük RMSE değerleriyle benzer şekiller ortaya çıkmaktadır (bakınız Şekil 3(a), 4(a), 5(a)). VTEC fark haritalarından görüldüğü gibi, BMARS ve SH modellemeleri arasındaki değişimler (Şekil 6(a)) B-Spline ve SH modellemeleri arasındaki değişimlerden (Şekil 7(a)) daha büyüktür. Çünkü BMARS, B-Spline'a dayalı modellemeye göre veriye daha iyi uyum göstermektedir. S1 veri grubunun değerlendirilmesinden sonra RMS değerlerine göre
BMARS ve B-Spline modellemesi benzer sonuçlar ortaya çıkarmaktadır; Şekil 6(a) da RMS $=0.8251$ TECU ve Şekil 7(a) da RMS $=0.7675$ TECU. S2 veri grubundaki VTEC değişimleri S1 grubundaki değişimlerden daha yüksektir ve $\mathrm{SH}$ modellemesinde daha yüksek RMSE değerine neden olmaktadır (Şekil 4(b)). Bu zaman aralığı içerisinde daha önce de değinilen jeomanyetik fırtına nedeniyle VTEC 30-35 TECU gibi yüksek bir değere ulaşmaktadır. Bunun sonucu olarak BMARS ölçek indeksini 4'e çıkarmaktadır. Algoritmanın ölçek indeksini arttırması BMARS'ın ürettiği yerel baz fonksiyonları sayısının B-Spline düzey 3 den daha fazla olduğunun göstergesidir. Bu fark aynı zamanda VTEC fark haritalarından da açıkca görülmektedir. S2 veri grubu için modellerin benzer VTEC haritaları üretmesine rağmen (Şekil 3(b), 4(b), 5(b)) özellikle BMARS ve SH modellemesi arasındaki fark (Şekil 6(b)) açık olarak sergilenmektedir. VTEC fark haritalarının nümerik sonuçları RMS cinsinden karşılaştırıldığında, Şekil 6(b) ve 7(b) de de görüldüğü gibi, B-Spline ve SH modellemesi arasındaki fark, BMARS ve SH modellemesi arasındaki farktan daha düşüktür. Bunun BMARS model davranışıyla açıklanabildiği ve modelin B-Spline baz fonksiyonlarından daha fazla sayıda yerelleştiren baz fonksiyonlarını içermesiyle ilgili olduğu düşünülmektedir. BMARS ve B-Spline modelleme sonuçları arasındaki benzeşmezlik her iki modeldeki baz fonksiyonlarının farklı bir şekilde üretilmesi ve farklı bir model yapılandırma stratejisine dayanmaktadır. Bu durum S2 veri grubu için Şekil 8 deki VTEC haritalarından açıkca gözlenmektedir. Üretilen tüm şekiller ve onların nümerik analizleri ışığında BMARS algoritmasının SH ve B-Spline modellemelerden daha küçük ve daha iyi RMSE değerleri ürettiği sonucuna varılmaktadır. Çünkü BMARS, bütün VTEC fark haritalarından da görüldüğü gibi, gözlemlere daha iyi uyum sağlamakta ve küçük ölçekli yerel özellikleri eklemektedir.

\section{Sonuçlar}

Bu çalışmada Çok Değişkenli Uyabilen B-Spline Regresyonu (BMARS) bölgesel VTEC'in modellenmesine uygulanmıştır. BMARS algoritmasının başarısı ve uyabilirliği 15 Şubat 2011 tarihindeki X-sınıfı güneş patlaması sonucu oluşan Jeomanyetik firtına esnasında Avrupa üzerindeki GPS istasyonlarından toplanan gerçek veriler kullanılarak gösterilmiştir. S1 ve S2 şeklinde olmak üzere iki alt grup veriyi içeren testlerin sonucunda BMARS algoritmasının bölgesel iyonosferik VTEC dağılımını etkin bir şekilde modelleme kabiliyetine sahip olduğu ortaya konulmuştur. SH, B-Spline ve BMARS modellemelerinde genelde bir bağdaşma olduğu gözlenmektedir. Bununla birlikte, BMARS'ın SH ve B-Spline algoritmalarıyla görsel ve nümerik karşılaştırılmaS1, BMARS'1n daha küçük RMSE değerleriyle daha yumuşak VTEC haritaları ürettiğini göstermiştir. $\mathrm{Bu}$ sonuçlara dayanarak BMARS algoritmasının bölgesel VTEC modellemesi için, gerçekte küresel fonksiyonlar sınıfına giren SH modellemesinden daha uygun olduğu tesbit edilmiştir. Diğer bir sonuç ise, BMARS'ın bölgesel B-Spline'a dayalı VTEC modelleme yöntemine karşın uygulanabilir bir alternatif algoritmayı teşkil etmesidir. 


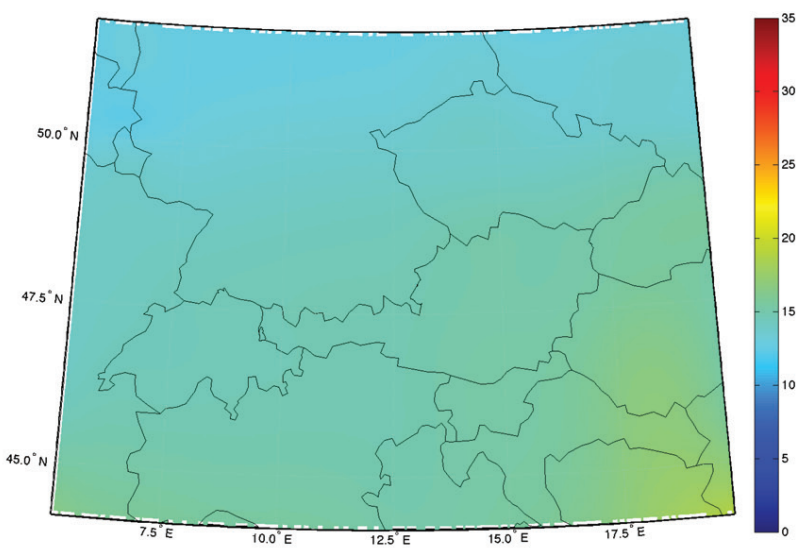

(a) 17 Şubat 2011, RMSE $=0.3825 \mathrm{TECU}, \quad$ seçilmiş terimlerin sayısı $=279, \mathrm{GCV}=0.1691$, ölçek indeksi $=3$

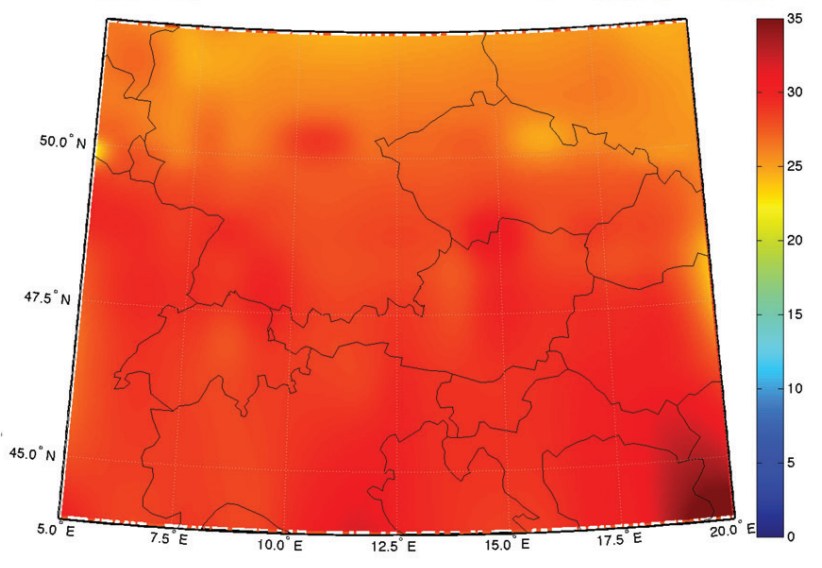

(b) 18 Şubat 2011, RMSE $=0.4601$ TECU, seçilmiş terimlerin sayısı $=289, \mathrm{GCV}=0.2913$, ölçek indeksi $=4$

Şekil 3: S1 ve S2 den saat 10:00 AM,UT için BMARS'tan üretilen VTEC haritaları. (a) RMSE =0.3825; (b) $R M S E=0.4601$.

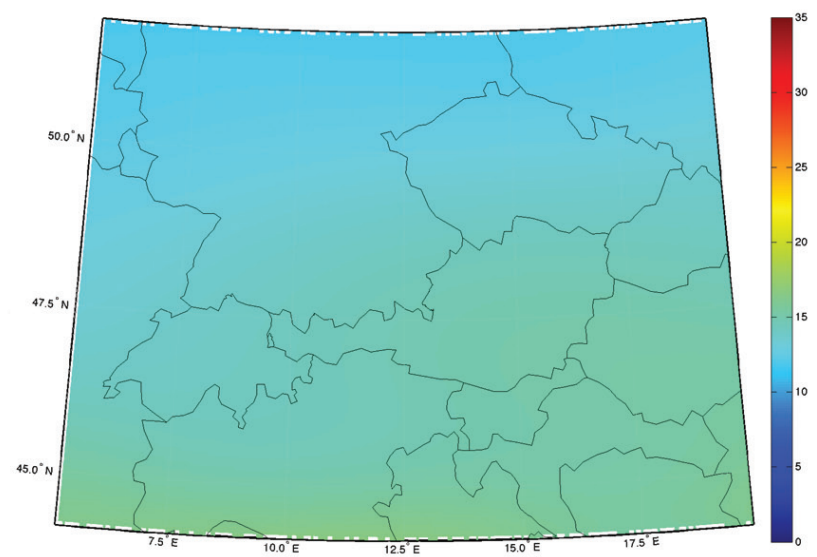

(a) 17 Şubat 2011, RMSE $=0.562$ TECU

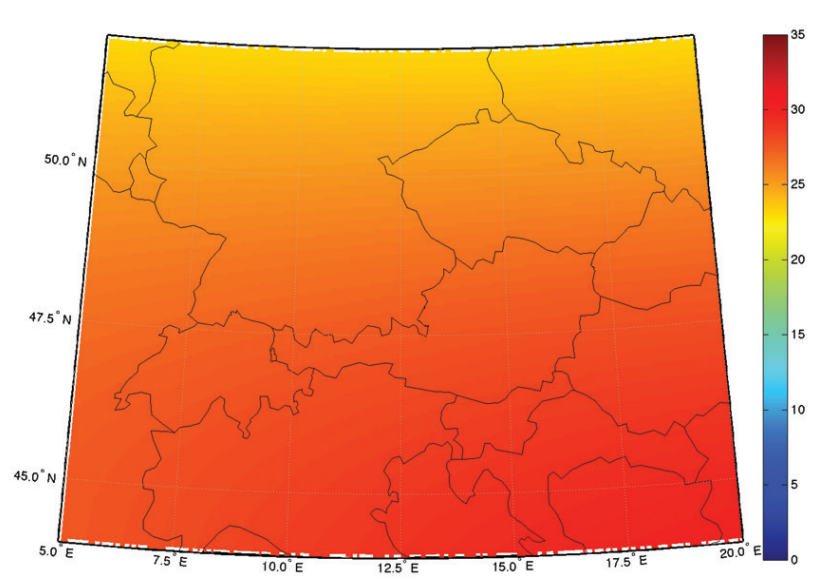

(b) 18 Şubat 2011, RMSE $=0.893$ TECU

Sekil 4: S1 ve S2 saat 10 AM,UT için derece, $n=8$ ve mertebesi, $m=8$ küresel harmonik açılımından hesaplanan VTEC haritaları. (a) RMSE $=0.562$ TECU; (b) RMSE = 0.893 .

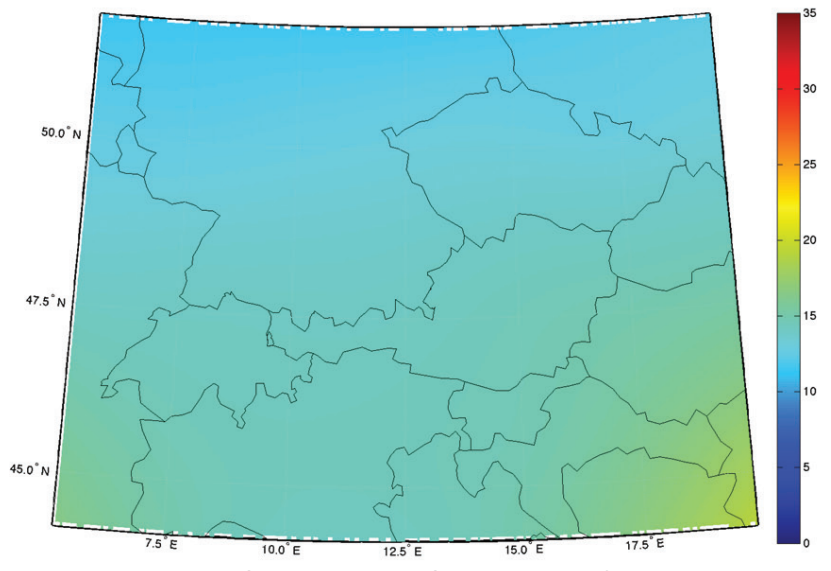

(a) 17 Şubat 2011, RMSE $=0.387$ TECU

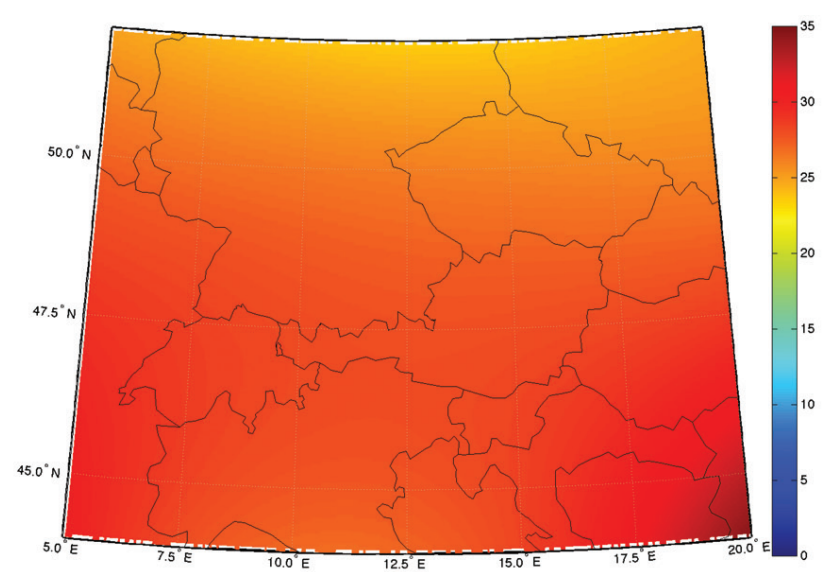

(b) 18 Şubat 2011, RMSE $=0.502$ TECU

Figure 5: S1 ve S2 saat 10 AM, UT için karesel 3D B-Spline düzey 3 modelinden (katsayilarin sayıSı $=1000$ ) hesaplanan VTEC haritaları. (a) RMSE = 0.387 TECU; (b) RMSE = $0.502 \mathrm{TECU}$. 


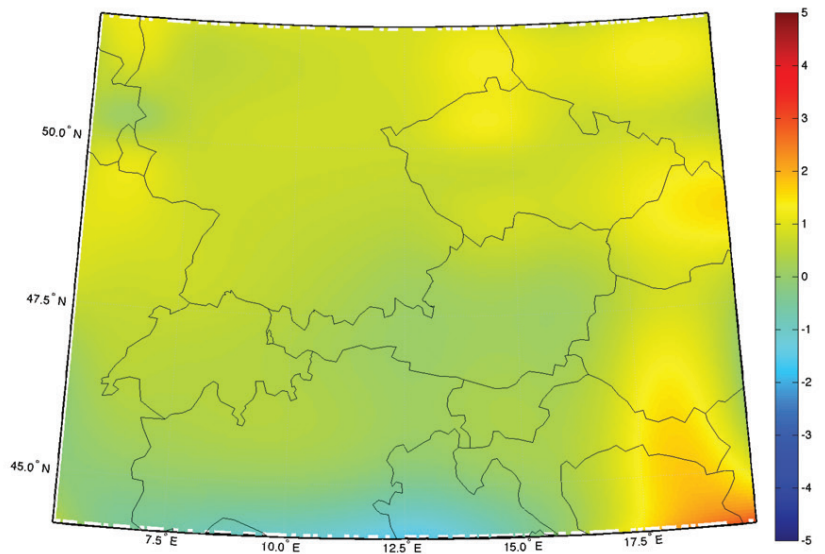

(a) 17 Şubat 2011; RMS $=0.8251$ TECU

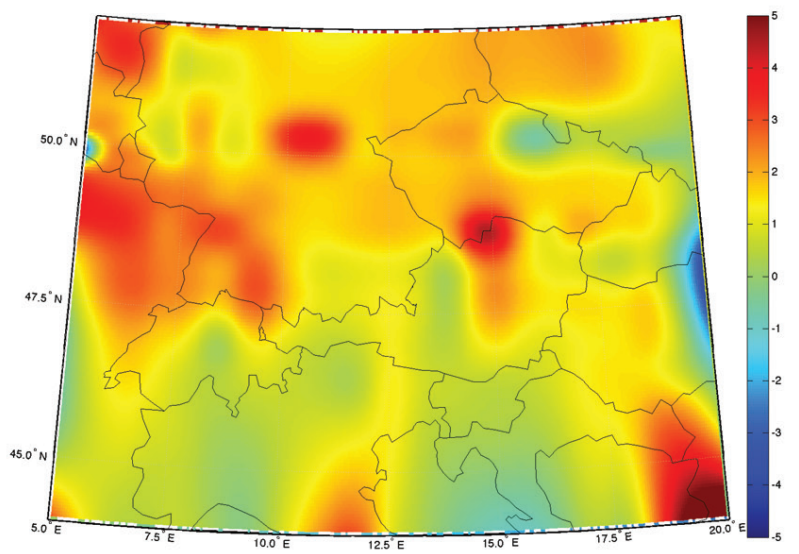

(b) 18 Şubat 2011; RMS $=1.7009$ TECU

Şekil 6: S1 ve S2 saat 10:00 AM,UT için BMARS ve SH modellerinin VTEC fark haritaları. (a) RMS =0.8251 TECU; (b) RMS = 1.7009 TECU.

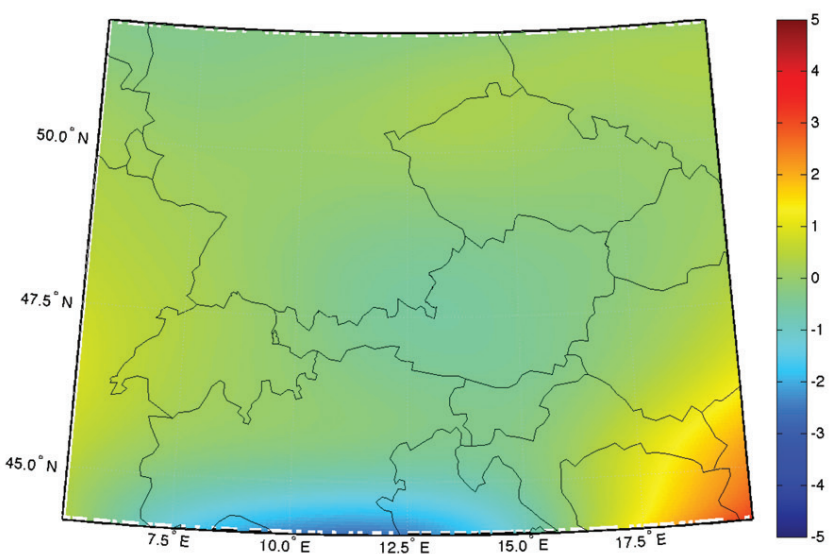

(a) 17 Şubat 2011; RMS $=0.6516$ TECU

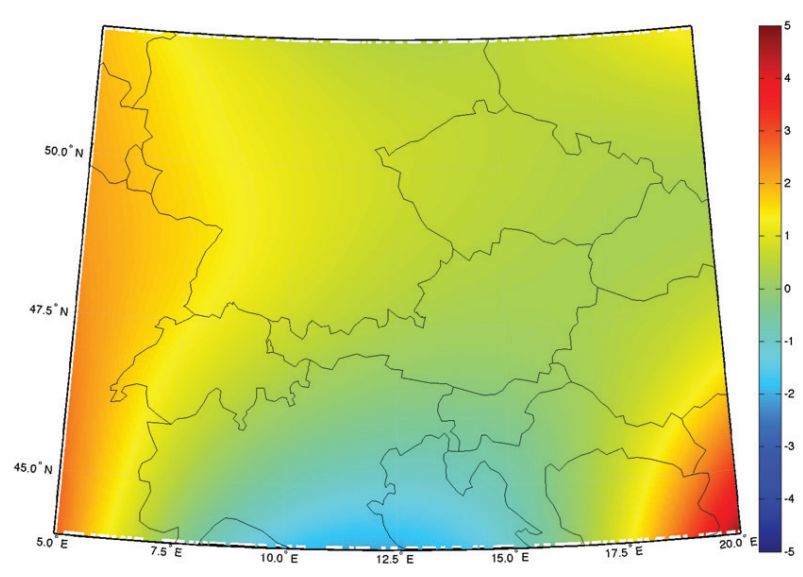

(b) 18 Şubat 2011; RMS=1.078 TECU

Şekil 7: S1 ve S2 saat 10:00 AM,UT için 3D karesel B-Spline ve SH modellerinin VTEC fark haritaları (a) RMS =0.6516 TECU; (b) RMS = 1.078 TECU.

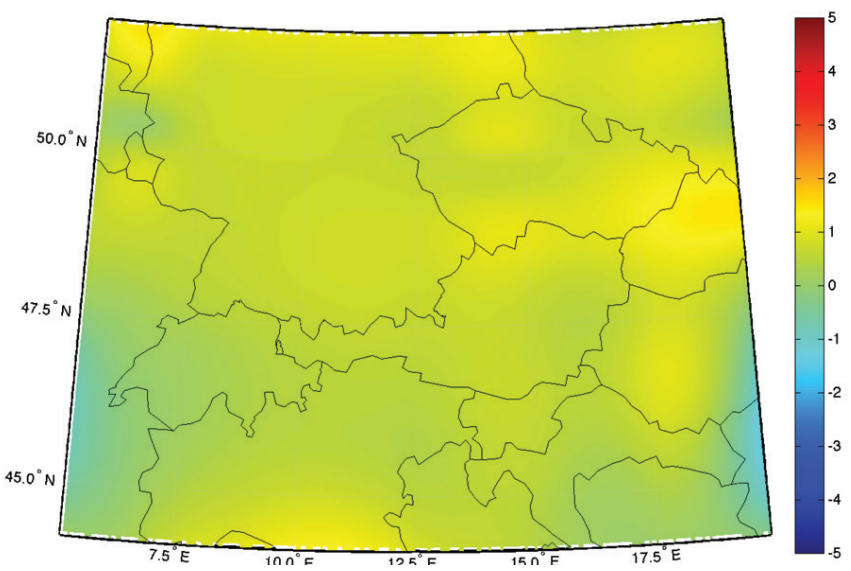

(a) 17 Şubat 2011; RMS $=0.7675$ TECU

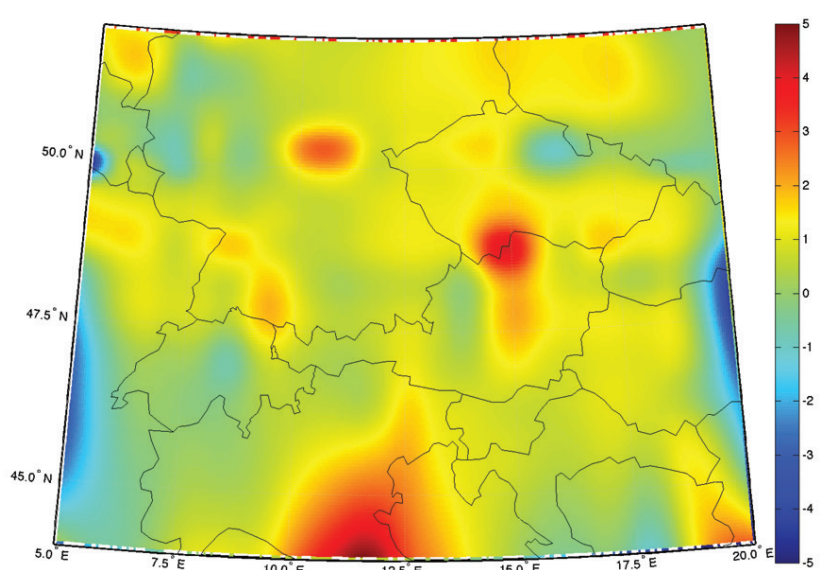

(b) 18 Şubat 2011; RMS $=1.0215$ TECU

Şekil 8: S1 ve S2 saat 10:00 AM,UT için BMARS ve B-Spline modellerinin VTEC fark haritaları. (a) RMS $=0.7675$ TECU, (b) RMS $=1.0215$ TECU 


\section{Kaynaklar}

Bakin S., Hegland M., Osborne M., (1997), Computational techniques and applications conference: Can mars be improved with b-splines?, Computational Techniques and Applications Conference, Adelaide, Avustralya.

Bakin S., Hegland M., Williams G., (2000), Mining taxation data with parallel BMARS, Parallel Algorithms and Applications, 15, 37-55.

Brunini C., Meza A., Azpilicueta F., Zele M.A.V., (2004). A new ionosphere monitoring technology based on GPS, Astrophysics and Space Science, 290(3-4), 415-429.

Brunini C., Camilion E., Azpilicueta F., (2010), Assessment of SIRGAS ionospheric maps errors based on a numerical simulation, EGU General Assembly, 2-7 Mayis, Vienna, Avusturya, s. 1840

Cox M., (1982), Practical spline approximation, Topics in Analysis' in İçinde, (Turner P., Ed.), Lecture Notes in Mathematics, Cilt. 965, Springer, Berlin/Heidelberg, ss.79-112.

Crino S., Brown D.E., (2007), Global Optimization with Multivariate Adaptive Regression Splines. Systems, Man, and Cybernetics, Part B: Cybernetics, IEEE Transactions on, 37(2), 333-340.

Dettmering D., (2003), The Utilisation of The GPS For Modelling the Ionosphere in Three Dimensions, Teknik Raporlar, Report Nr. 2003.1, Doktora Tezi, Department of Geodesy and and Geoinformatics, Stuttgart Üniversitesi.

Durmaz M., Karslioglu M.O., Nohutcu M., (2010), Regional VTEC modeling with multivariate adaptive regression splines, Advances in Space Research, 46(2), 180-189.

Ekman T., Kubin G., (1999), Nonlinear prediction of mobile radio channels: measurements and mars model designs, IEEE International Conference on Acoustics Speech and Signal Processing, 5, 2667-2670.

Friedman J.H., (1991), Multivariate adaptive regression splines, The Annals of Statistics, 19(1), 1-67.

Garcia-Fernandez M., (2004), Contributions to the 3D Ionospheric Sounding with GPS Data. Doktora Tezi, Department of Applied Mathematics IV and Applied Physics, Technical University of Catalonia, Barcelona, İspanya.

Hernandez-Pajares M., Sanz J.M.J.J., (1999), New approaches in global ionospheric determination using ground GPS data, Journal of Atmospheric and Solar Terrestrial Physics, 61(16), 1237-1247.

Hofmann-Wellenhof B., Lichtenegger H., Wasle E., (2008), GNSS Global Navigation Satellite Systems GPS, GLONASS, Galileo more. Springer, Avusturya.

Jin S.G., Luo O., Park P., (2008), GPS observations of the ionospheric F2-Layer behaviour during the 20th November 2003 geomagnetic storm over South Korea, Journal of Geodesy, 82(12), 883-892.

Jin S.G., Park J., Wang J., Choi B., Park P., (2006), Electron density profiles derived from ground-based GPS observations, Journal of Navigation, 59(3), 395-401.

Jin S.G., Wang H.P., Zhu W.Y., (2004), Realtime prediction and monitoring of the total ionospheric electron content by means of GPS observations, Chinese Astronomy and Astrophysics, 28(3), 331-337.
Lewis P.A.W., Stevens J.G., (1991), Nonlinear modeling of time series using Multivariate Adaptive Regression Splines (MARS), Journal of the American Statistical Association, 86(416), 864877.

Liu Z., Gao Y., (2004), Ionospheric TEC Predictions over a local area GPS reference network, GPS Solutions, 8(1), 23-29.

Mannucci A.J., Wilson B.D., Yuan D.N., Ho C.H., Lindqwister U.J., Runge T.F., (1998), A global mapping technique for GPSDerived ionospheric total electron content measurements, Radio Science, 33(3), 565-582.

Misra P., Enge P., (2003), Global Positioning System: Signals, Measurements, and Performance. Ganga-Jamuna Press, Massachusetts, ABD.

Nohutcu M., Karslioglu M., Schmidt M., (2010), B-Spline modeling of VTEC over Turkey using GPS observations, Journal Of Atmospheric and Solar- Terrestrial Physics, 72(7-8), 617-624.

Nohutcu M., Karslioglu M.O., Gucluer B., Schmidt M., Zeilhofer C., Zhang Z., Ergintav S., (2007), Local modeling of TEC using GPS observations, TUJK Scientific Meeting Proceedings'in İçinde, Ankara, Türkiye.

Policy Workshop Report, (2011), Satellite Navigation \& Space Weather: Understanding the Vulnerability \& Building Resilience, American Meteorological Society.

Schaer S., (1999), Mapping and Predicting the Earth's Ionosphere Using the Global Positioning System, Doktora Tezi, University of Bern , İsviçre.

Schmidt M., (2007), Wavelet modeling in support of IRI, Advances in Space Research, 39(5), 932-940.

Schmidt M., Bilitza D., Shum C.K., Zeilhofer C., (2007a), Regional 4-D Modeling of the ionospheric electron content, Advances in Space Research, 42(4), 782-790.

Schmidt M., Karslioglu M.O., Zeilhofer C., (2007b), Regional multi-dimensional modeling of the ionosphere from satellite data, TUJK Scientific Meeting Proceedings'in İçinde, Ankara, Türkiye.

Seeber G., (2003), Satelite Geodesy, de Gruyter, Berlin, Almanya, 589 ss.

Wielgosz P., Grejner-Brzezinska D.A., Kashani I., (2003), Regional ionosphere mapping with kriging and multiquadric methods, Journal of Global Positioning Systems, 2(1), 48-55.

Yang C.C., Prasher S.O., Lacroix R., Kim S.H., (2004), Application of Multivariate Adaptive Regression Splines (MARS) to simulate soil temperature. Transactions of the ASAE, 47(3), 881-887.

Yuan Y., Ou J., (2002), Differential areas for differential stations (DADS): A new method of establishing grid ionospheric model, Chinese Science Bulletin, 47(12), 1033-1036.

Zeilhofer C., (2008), Multi-Dimensional B-Spline Modeling of Spatio-Temporal Ionospheric Signals, German Geodetic Commission, Cilt.123, Seri A, Muenchen. 